\title{
The plasmasphere during a space weather event: first results from the PLASMON project
}

János Lichtenberger ${ }^{1,2, *}$, Mark A. Clilverd ${ }^{3}$, Balázs Heilig ${ }^{4}$, Massimo Vellante ${ }^{5}$, Jyrki Manninen ${ }^{6}$, Craig J. Rodger ${ }^{7}$, Andrew B. Collier ${ }^{8}$, Anders M. Jørgensen ${ }^{9}$, Jan Reda ${ }^{10}$, Robert H. Holzworth ${ }^{11}$, Reinhard Friedel ${ }^{12}$, and Mea Simon-Wedlund ${ }^{7}$

1 Space Research Group, Department of Geophysics and Space Sciences, Eötvös University, Pázmány P. sétány 1/A, H-1117 Budapest, Hungary

*Corresponding author: e-mail: Iityi@sas.elte.hu

2 Geodetic and Geophysical Institute, Research Center for Astronomy and Earth Sciences, Hungarian Academy of Sciences, Csatkai u. 6-8, H-9400 Sopron, Hungary

3 British Antarctic Survey, High Cross, Madingly Road, CB3 0ET Cambridge, UK

4 Geological and Geophysical Institute of Hungary, Stefnia t 14, H-1143 Budapest, Hungary

5 Physics Department, University of L'Aquila Via Vetoio, I-67010 Coppito-L'Aquila, Italy

6 Sodankylä Geophysical Observatory Tähteläntie 62, 99600 Sodankylä, Finland

7 Department of Physics, University of Otago, P.O. Box 56, 9054 Dunedin, New Zealand

8 SANSA Space Science, P.O. Box 32, 7200 Hermanus, South Africa; School of Physics, University of KwaZulu-Natal, 4001 Durban, South Africa

9 Electrical Engineering Department, New Mexico Institute of Mining and Technology, 801 Leroy Place, NM 87801 Soccoro, USA

10 Institute of Polish Academy of Sciences, 05-622 Belsk Duzy, Poland

11 Department of Earth and Space Sciences, University of Washington, 4333 Brooklyn Ave NE, 98195-9472 Seattle, USA

12 Los Alamos National Laboratory, P.O. Box 1663, NM 87545 Los Alamos, USA

Received 1 November 2012 / Accepted 14 June 2013

\begin{abstract}
The results of the first 18 months of the PLASMON project are presented. We have extended our three, existing ground-based measuring networks, AWDANet (VLF/whistlers), EMMA/SANSA (ULF/FLRs), and AARDDVARK (VLF/perturbations on transmitters' signal), by three, eight, and four new stations, respectively. The extended networks will allow us to achieve the four major scientific goals, the automatic retrieval of equatorial electron densities and density profiles of the plasmasphere by whistler inversion, the retrieval of equatorial plasma mass densities by EMMA and SANSA from FLRs, developing a new, data assimilative model of plasmasphere and validating the model predictions through comparison of modeled REP losses with measured data by AARDDVARK network. The first results on each of the four objectives are presented through a case study on a space weather event, a dual storm sudden commencement which occurred on August 3 and 4, 2010.
\end{abstract}

Key words. plasmasphere - whistler - FLR - data assimilation - relativistic electron precipitation

\section{Introduction}

The PLASMON FP7-Space project (A new, ground-based data assimilative model of the Earth's Plasmasphere - a critical contribution to Radiation Belt modeling for Space Weather purposes, http://plasmon.elte.hu) addresses space weather models to improve specification and prediction capabilities, with emphasis on the linkage of the different physical processes that occur simultaneously or sequentially in many domains such as the ionosphere, plasmasphere, and radiation belts. The project started on February 1, 2011 and is expected to be completed on July 31,2014 . In this paper we describe the PLASMON project, and report on progress in the first two years of the project. We also present an example of how the scientific work-packages link together to produce greater understanding of plasmaspheric dynamics and the influence of this upon the radiation belts. The project consists of four major objectives, described below:
1. Automatic retrieval of equatorial electron densities and density profiles by the Automatic Whistler Detector and Analyzer Network (AWDANet). Recently Eötvös University has developed a new, experimental Automatic Whistler Detector and Analyzer (AWDA) system (Lichtenberger et al. 2008) that is capable of detecting whistlers; we use this system to process lightning-generated whistlers with no human interaction. AWDANet is evolving and now covers low, mid, and high magnetic latitudes with wide longitudinal coverage. Recent developments in whistler inversion methods for multiple-path whistler groups propagating at mid and high latitude (Lichtenberger 2009) will allow us to retrieve electron density profiles automatically for a wide range of $L$-values. In the project, the AWDANet has been extended to have better spatial and temporal coverage and thus is able to provide density profiles for different MLTs which can be used as a data source for space 
weather models. The implementation of the new automatic whistler analyzer (AWA) method (Lichtenberger et al. 2010) has been installed in AWDANet nodes. The transformation of AWDANet to work in quasi-real-time mode of operation is in progress.

2. Retrieval of equatorial plasma mass densities by the Europen quasi-Meridional Magnetometer Array (EMMA) and cross-calibration of whistler and Field Line Resonance (FLR) methods for determining electron and mass density, respectively. The goal of the EMMA, which is created from SEGMA (South European GeoMagnetic Array); Vellante et al. (2004), and MM100 (Magnetic Meridian 100) arrays (Heilig et al. 2010) in the project is to monitor the equatorial plasma mass density based on the detection of geomagnetic FLRs. None of the earlier monitoring systems, however, were "space weather" operational in the sense that they never produced quasireal-time products. The latitude coverage was also not sufficient to monitor the whole plasmasphere. In contrast to the whistler method the FLR method can be used to infer the plasma mass density even in the plasmatrough and to also identify the location of the plasmapause. We have unified the isolated European efforts to call into being a joint European network, EMMA, with stations ranging from Italy to the northern Finland ( $L$-shells 1.6-6.7). We use and upgraded existing magnetometer networks (IMAGE), which were originally established for other purposes and other requirements (resolution, sampling rate, timing), but the data of which are exploited for plasmasphere observations as well. In accordance with these goals we will:

(a) Unify and extend the SEGMA, MM100, and IMAGE networks into EMMA;

(b) Develop an approach to allow automatic FLR identification and FLR inversion to estimate mass densities;

(c) Develop all EMMA stations to work in quasi-real-time modes of operation and evaluate relative abundances of heavy ions in the plasma composition from simultaneous determinations of mass density (FLR method) and electron density (whistler method).

3. Data assimilative modeling of the Earth's plasmasphere. Even dense measurements only sample the plasmasphere at limited resolution in both space and time. Yet determining the effect of wave-particle interactions on the radiation belts requires a continuous map of the plasma density in both time and space. In order to provide such a complete map it becomes necessary to interpolate between measurements, again in both time and space, with data assimilation schemes to combine plasmaspheric measurements with a numerical physics-based plasmasphere model. The two data assimilation schemes which we are pursuing are Ensemble Kalman filtering (Evensen 2003) and particle filtering (Nakano et al. 2008).

4. Modeling Relativistic Electron Precipitation (REP) losses from the radiation belts using the Antarctic-Arctic Radiation-belt (Dynamic) Deposition-VLF Atmospheric Research Konsortium (AARDDVARK) network. During a geomagnetic storm the length of time during which space assets are in danger is determined by the efficiency of the radiation belt loss mechanisms, particularly through REP into the atmosphere. We use the assimilative model of the plasmasphere to identify regions where plasmaspheric structures such as the regions occurring on, inside, and outside of the plasmaspause and/or composition changes are likely to result in enhanced electron losses. We will monitor the occurrence and properties of REP using the ground-based AARDDVARK network (Clilverd et al. 2009), which will be extended during the project to have better spatial and temporal coverage.

At the end of the project we will provide real-time data of plasmaspheric densities, a data-assimilative model of the plasmasphere and a model of REP losses. All these data, models and information will significantly contribute to European capacity to estimate and prevent damage of space assets from space weather events as well as to improving forecasting and prediction of disruptive space weather events.

One network (AWDANet) measures Very Low Frequency (VLF) waves to capture and analyze whistlers, another network (EMMA) measures Ultra Low Frequency (ULF) signals to capture and analyze FLR. Methods based on the two phenomena are capable of providing plasmaspheric densities. The two methods are complementary to each other due to the spatial and temporal occurrences of whistlers and FLRs. Overlapping events (which mostly at late afternoon and early morning times) from the two techniques are used for cross-calibration of the methods. New opportunities are available with the launch of Van Allen Probes NASA mission (http://www.nasa.gov/ vanallenprobes/), we plan to use in-situ density and wave measurements to calibrate the two ground-based methods independently, overcoming the lack of common events in space and time. Monitoring of the plasmasphere by whistlers and FLRs is the basic objective of this proposal, while the third (dataassimilative modeling of the Earth's plasmasphere) uses these data to provide a high-fidelity model. The fourth objective (identifying electron loss to the atmosphere from the different regions of the plasmasphere) demonstrates one application of the new plasmasphere model in providing value added information on the loss processes for use in radiation belt models making use of measurements by a third ground-based network (AARDDVARK).

In the following, we discuss the techniques in more detail, presenting for illustration results on a space weather event, a dual storm sudden commencement (SSC) which occurred on August 3 and 4, 2010, revealing how the four major objectives of PLASMON contribute to the analysis of an event.

\section{Automatic retrieval of equatorial electron densities and density profiles by AWDANet}

The cold electron density distribution of the plasmasphere cannot be easily measured routinely, but is a key parameter for modeling of the plasmasphere and radiation belts. Whistlers have been regarded as cheap and effective tools for plasmasphere diagnostics since the early years of whistler research (e.g., Sazhin et al. 1992), but did not become a real operational tool since reducing whistler data to equatorial densities was very labour intensive. Recently the Space Research Group of Eötvös University has developed a new, experimental AWDA system that is capable of detecting whistlers and we use this system to process lightning-generated whistlers with no human interaction. The AWDA system consists of two major blocks: the automatic whistler detector (AWD) and automatic whistler analyzer (AWA). The former works in real time and is able 

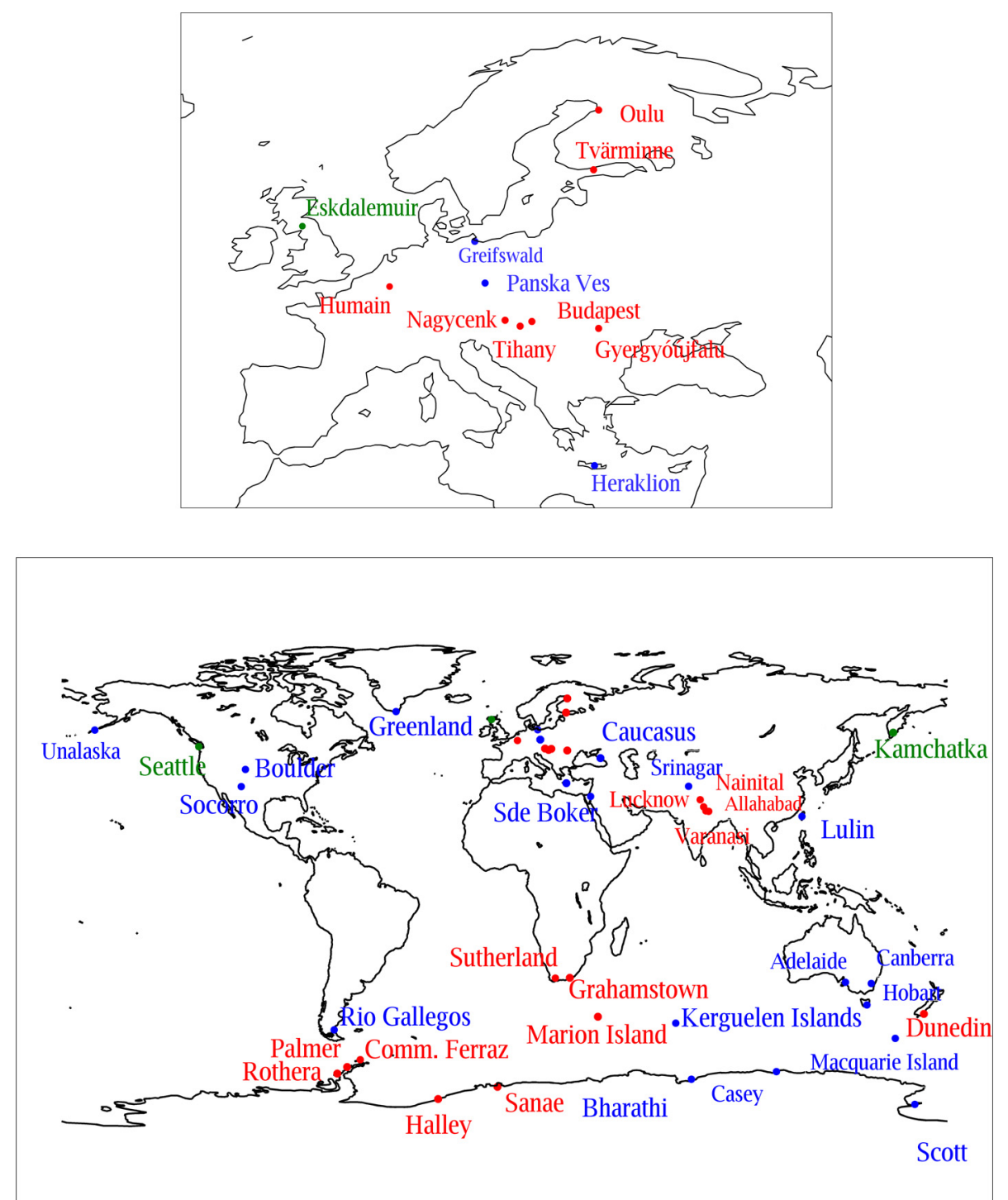

Fig. 1. AWDANet stations. Top panel: European stations. Bottom panel: Global stations. Names in red are operational, names in blue are planned stations. Names in green are the three new stations installed in the PLASMON project.

to detect whistlers in the raw VLF data stream, saving into disk files only those sections of the input stream that contains whistlers. The latter block takes the saved files and infers equatorial electron densities and propagation paths. A global network formed by AWDA systems (AWDANet) is evolving and now covers low, mid and high magnetic latitudes (Lichtenberger et al. 2008). In PLASMON, AWDANet has been extended with three new stations to have latitudinal and longitudinal coverage that are close to optimal. The three new stations are: Eskdalemuir (Scotland), Forks (Seattle, USA) and Karymshina (Kamchatka, Russia) (Fig. 1).

A recent development in whistler inversion methods for multiple-path whistler groups propagating on mid and high latitude (Lichtenberger 2009) allows us to retrieve electron density profiles automatically for a wide range of $L$-values. The inversion methods used for whistlers on mid and high latitude paths are now being used for low latitude whistlers as well. In PLASMON, we have developed the implementation of the AWA method (Lichtenberger et al. 2010) on normal CPU computers, while the implementation on Graphical Processing Unit (GPU)based parallel processing units is going on. The final goal is to achieve a quasi-real-time mode of operation. With this mode of operation, a node on the AWDANet system will be able to provide 10-15 equatorial electron densities or density profiles per hour by processing multiple-path whistler groups, which is enough to monitor the changes in the plasmasphere caused either by MLT changes or the dynamics of the magnetosphere itself (e.g., Darrouzet et al. 2009). However, it has to be noted, that the number and/or quality of detected whistlers may not allow the inversion of $10-15$ whistler events per hour. In this case, a plasmasphere model can be used to fill the gaps, complemented with electron densities obtained from another AWDANet station (different magnetic latitude and longitude).

The enhanced global coverage of AWDANet allows us to record whistlers in wide $L$-range, from: 1.2 (at Indian stations) to $>5$ (at Arctic and Antarctic stations). The inversion algorithm, however, can be applied for whistlers propagate on $L>1.4$, due to the validity of the field-aligned density model. The upper limit varies with the position of the plasmapause. We plan to use data from all (i.e., global) stations. The typical errors of equatorial electron densities obtained from the automated whistler analysis can be between $1 \%$ and $50 \%$, depending on the quality of traces in whistler events (sharpness, frequency coverage, signal to noise ratio). 


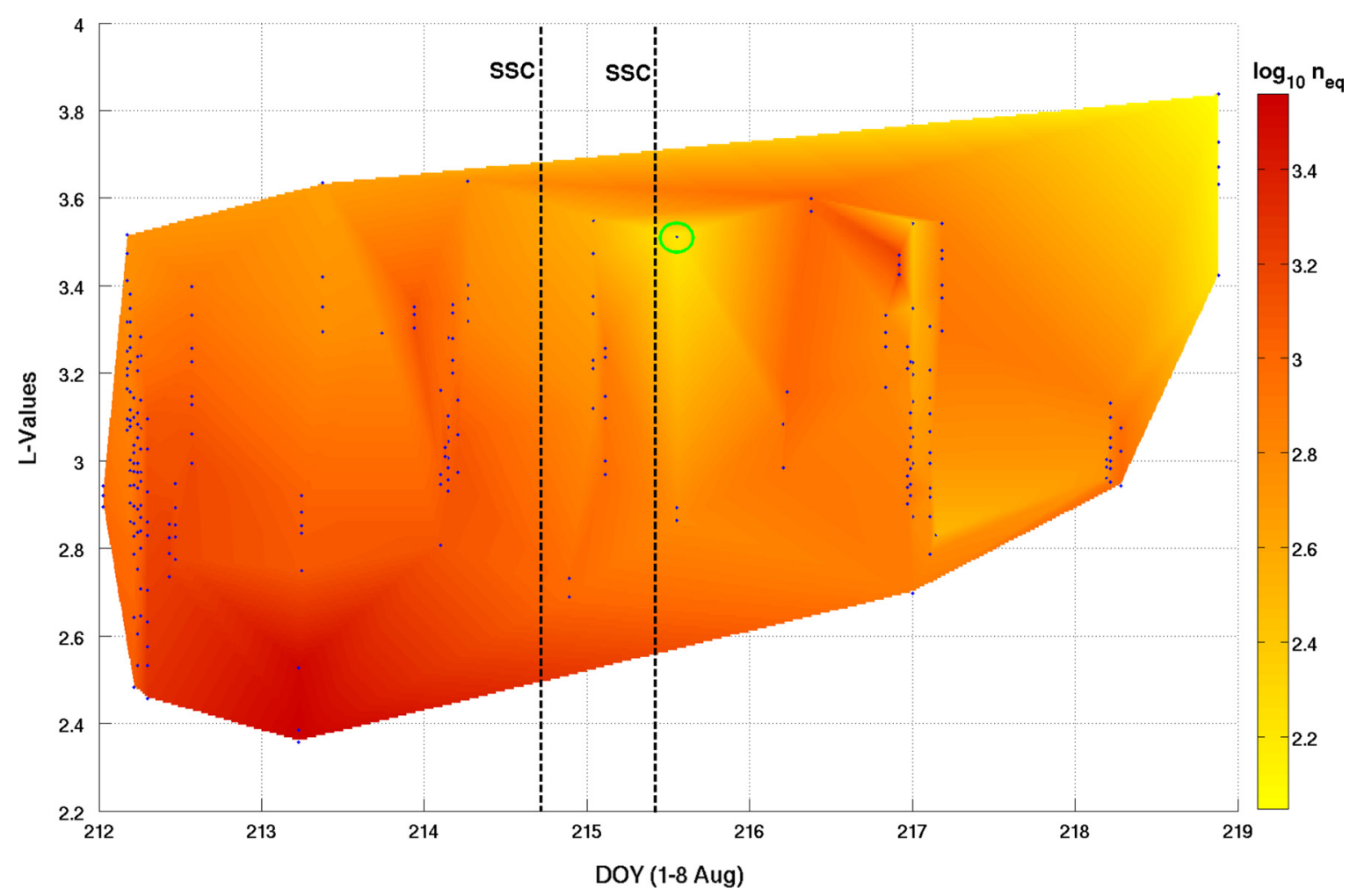

Fig. 2. A contour map of equatorial electron density variation between August 1 and 8, 2010. The blue dots are the whistler traces identified in the 41 events processed. The trace in the green circle is a knee whistler.

Table 1. The calculated $L$-value, equatorial electron density, tube electron content, and estimated coupling flux (electron refilling rate) for six whistler traces. The tube contents and coupling fluxes are referenced to $1000 \mathrm{~km}$. See details in the text.

\begin{tabular}{|c|c|c|c|c|}
\hline Time & $L$-value & $n_{\mathrm{eq}}\left(\mathrm{cm}^{-3}\right)$ & $N_{T}\left(10^{13} \mathrm{el} \mathrm{cm}^{-2}-\right.$ tube $)$ & (Ф) $\left(10^{7} \mathrm{el} \mathrm{cm}^{-2} \mathrm{~s}^{-1}\right)$ \\
\hline 2010-08-01UT05:44:37.687 & $2.96 \pm 0.01$ & $1538 \pm 16$ & $4.37 \pm 0.07$ & - \\
\hline 2010-08-02UT05:54:13.975 & $2.83 \pm 0.05$ & $1737 \pm 110$ & $4.00 \pm 0.37$ & $-4.32 \pm 4.38$ \\
\hline 2010-08-03UT04:55:10.149 & $2.98 \pm 0.02$ & $1859 \pm 29$ & $5.40 \pm 0.16$ & $16.93 \pm 4.92$ \\
\hline 2010-08-05UT04:54:45.673 & $2.93 \pm 0.00$ & $1317 \pm 4$ & $3.63 \pm 0.02$ & $-10.26 \pm 0.94$ \\
\hline 2010-08-06UT04:23:04.612 & $2.93 \pm 0.00$ & $1177 \pm 5$ & $3.28 \pm 0.02$ & $-4.13 \pm 0.35$ \\
\hline 2010-08-07UT04:37:21.844 & $2.98 \pm 0.01$ & $534 \pm 2$ & $1.76 \pm 0.02$ & $-17.3 \pm 0.39$ \\
\hline
\end{tabular}

To test the implemented AWA method, we chose 83 whistler events covering the period August 1-7, 2010, that is the period prior and after the dual-SSC. The AWDA sytem recorded almost 2600 events in this period at Dunedin (New-Zealand). The time-distribution of these events is not even, because the occurrence of the events is far too varied to produce an even distribution, as is commonly the case at this location (Collier et al. 2010). Our 83 whistler events include single whistlers and whistler groups as well. All these events were processed by the AWA algorithm and the analysis of 41 was completed with sufficiently high-quality thresholds (see Lichtenberger et al. 2010). These 41 detected whistler events contain 224 whistler traces. The result of an inversion of a whistler event is the $A$ and $B$ parameters and the $L$-values of the identified traces in the event, producing an automatic "scaling" of the whistler traces. $A$ and $B$ are parameters to describe the $L$-dependence of the equatorial electron density (Lichtenberger 2009):

$$
\log _{10} n_{\mathrm{eq}}=A+B L, \quad 1.4<L<8 .
$$

$n_{\text {eq }}$ is then calculated for each $L$.
The goal of this case study was to test and tune the AWA algorithm. Figure 2 shows a contour map created from the all $\left(L, n_{\text {eq }}\right)$ pairs using Delaunay triangulation to fill the gaps between the scattered data points. It has to be noted, that this interpolation introduced artifacts due to the highly uneven distribution of data points. Though the gaps between the time of events prevent us from fully following the equatorial electron density variations during the study period, a slight (factor of 2) decrease can be seen after the first SSC and a more articulated decrease (factor of 3 ) after the second one around $L=3.5$. The data point in the green circle is a knee whistler, propagating at the plasmapause at $L=3.51$ where the equatorial electron density is $n_{\mathrm{eq}}=152 \mathrm{~cm}^{-3}$.

Though the data points are highly uneven both in space and time, we found six whistler traces propagating approximately along the same field line about 1-2 days apart. Thus the events occur on the same $L$-shell and MLT and we can calculate the coupling fluxes, i.e., electron refilling rates in five cases. The events are shown in Table 1. The first three events were recorded before the SSCs, while the last three ones after the SSCs. Each event was recorded at late afternoon in local time. 
The coupling flux $\Phi$ is calculated as $\left(N_{\mathrm{T}, 2}-N_{\mathrm{T}, 1}\right) /\left(t_{2}-t_{1}\right)$, where $t_{1}$ and $t_{2}$ are the time of the two consecutive events, $N_{\mathrm{T}, 1}$ and $N_{\mathrm{T}, 2}$ are the tube electron contents (see e.g., Park 1972) calculated at the same time. The tube electron content did not practically change between August 1 and 2, indicating a condition of saturated flux tube - the small negative flux is probably due to the difference in the two $L$-shells (2.96 vs. 2.83) used for the calculation. The flux is relatively high between August 2 and $3\left(16.93 \times 10^{7} \mathrm{el} \mathrm{cm}^{-2} \mathrm{~s}^{-1}\right)$ showing refilling of the flux tubes and negative between August 3 and $5\left(-10.26 \times 10^{7} \mathrm{el} \mathrm{cm}^{-2} \mathrm{~s}^{-1}\right)$, this interval spans over two days and includes the SSCs when the plasmasphere was eroded. However, both the last two coupling fluxes are negative, $-4.13 \times 10^{7} \mathrm{el} \mathrm{cm}^{-2} \mathrm{~s}^{-1}$ between August 5 and 6 and $-17.3 \times 10^{7} \mathrm{el} \mathrm{cm}^{-2} \mathrm{~s}^{-1}$ between August 6 and 7 suggesting prolonged erosion of the plasmasphere after the storms in the dusk sector at $L=\sim 2.9$.

\section{Retrieval of equatorial plasma mass densities by EMMA and SANSA magnetometer arrays}

Thanks to recent developments in magnetometry (e.g., reduction of noise), data acquisition (improved resolution and timing), and the theory (wave propagation, event detection, models, inversion) of magnetohydrodynamic (MHD) waves, the routine monitoring of the cold plasma mass density of the plasmasphere has become possible. EMMA was established in 2012 within the frame of PLASMON with the main goal to monitor the plasmaspheric mass density based on the detection of FLRs. EMMA was born through the unification and extension of previously existing European magnetic arrays: SEGMA (South European GeoMagnetic Array) (Vellante et al. 2004) and MM100 (Heilig et al. 2010) including the Finnish stations of IMAGE. At the end of 2012 EMMA consists of 25 stations (top panel, Fig. 3) from north Finland to Italy ( $L$-shells 1.6-6.7) as a joint effort of FMI (Finland), IGFPAS (Poland), SAS (Slovakia), MFGI (Hungary), and University of L'Aquila (Italy). PLASMON also has a smaller magnetometer network maintained by SANSA at South-African conjugate area (bottom panel, Fig. 3).The SANSA observations (SUT-HER) will allow examination of possible effects of north-south ionospheric asymmetries and will give independent estimates of the plasma mass density at $L=1.8$, therefore providing a check on the accuracy of the method. In addition, measurements from the new pair TSU-WBP will allow extension of the monitoring to a lower $L$-shell $(\sim 1.4)$. The instrumentation is similar at all sites. Low noise (mostly fluxgate) magnetometers are sampled with high resolution, samples are synchronized to GPS PPS signals. Data are transferred to the project servers for processing through the internet every $15 \mathrm{~min}$.

The first step of the EMMA data processing is the detection of FLRs. This is done by applying the phase gradient technique (Waters et al. 1991) on magnetic data recorded at two closely spaced $(100-300 \mathrm{~km})$ stations which are located along nearly the same magnetic meridian. In the dynamic cross phase spectra the FLR frequency shows up as the maximum of the phase difference between the two signals. Another characteristic feature of FLRs is the variation of the amplitude ratio of the two signals across the resonant frequency $\left(f_{\mathrm{FLR}}\right)$. The ratio is around 1 at $f_{\text {FLR }}$ and has a local minimum/maximum below/above this frequency. $f_{\mathrm{FLR}}$ is determined by the maximum in phase difference and the proximity of the amplitude ratio to one. An automated algorithm, FLRID (Field Line Resonance Identification) is being developed in PLASMON to do this job. FLRID also checks other parameters, such as the location of the inflection point in the amplitude ratio spectrum, the amplitude ratio at the inflection point, the magnitude of the phase difference, etc., that all help to identify the FLR frequency. These parameters also allow to estimate the uncertainty in the detected FLR frequency (Berube et al. 2003) and the resonance width (Green et al. 1993). Figure 4 shows three examples from different latitudes for the cross phase spectra that FLRID is based on, for August 1, 2010 (prior to the ssc). FLR frequencies are identified by the reddish horizontal stripes standing out from the greenish background from $\sim 04$ UT to $\sim 16$ UT at $\sim 10 \mathrm{mHz}$ (MEK-NUR, $L=3.7$ ), $\sim 15 \mathrm{mHz}$ (NUR-TAR, $L=3.2$ ), and $\sim 25 \mathrm{mHz}$ (SUW-BEL, $L=2.4$ ), respectively.

The inversion of the FLRs is possible if the magnetic field and the density distribution along the field line are known. Our inversion code, FLRINV, solves the MHD wave equation of the resonance (Singer et al. 1981) in an arbitrary magnetic field topology to infer the plasma mass density at the magnetic equatorial point of the field line. At the current stage of the development the T01 model (Tsyganenko 2002a, 2002b) is used to describe the magnetic field topology, while the field-aligned density distribution applied is a simple power-law distribution. However, at low latitudes more realistic distributions should be used because of the presence of heavy ions.

The typical uncertainties in the inferred equatorial plasma mass densities, which derive from the uncertainty in determining the FLR frequency, are of the order of $15-20 \%$. Additional uncertainties, which might be of the same order, can derive from the adopted field-aligned mass density distribution at low $(<2) L$-shells (Vellante \& Förster 2006), and from the magnetic field model used at high $L$-shells (Berube et al. 2006).

Figure 5 shows the plasma mass densities inferred from the available ULF measurements for the period August 1-8, 2010. The results indicate at the outermost $L$-shell a significant depletion (a factor 3 ) after the first SSC and a further decrease (a factor 10 with respect to the pre-storm conditions) after the second SSC. The strong depletion observed at this $L$-shell is in agreement with the expected position of the plasmapause (discussed later on in Fig. 8) which indicates that the flux tube at $L=3.7$ was outside the plasmasphere during the whole DoY 215. On August 8 (DoY 219) the density has completely recovered (i.e., 5 days after the first SSC).

The refilling between DoY 216 and DoY 219 (considering the density values at noon) took place at a rate of $83 \pm 20 \mathrm{amu} \mathrm{cm} \mathrm{cm}^{-3} \mathrm{~d}^{-1}$ which is equivalent to a net upward ion flux of $(7.3 \pm 1.7) \times 10^{7} \mathrm{amu} \mathrm{cm} \mathrm{cm}^{-2} \mathrm{~s}^{-1}$ across the $1000 \mathrm{~km}$ level. This value is in line with previous estimates obtained from day-to-day variations of flux tube content during the recovery phase of magnetic storms. Indeed, Chi et al. (2000) obtained $\Phi=6 \times 10^{7} \mathrm{amu} \mathrm{cm} \mathrm{cm}^{-2} \mathrm{~s}^{-1}$ at $L=2$ and Park (1970) obtained $\Phi=6 \times 10^{7} \mathrm{el} \mathrm{cm}^{-2} \mathrm{~s}^{-1}$ at $L=4$. Note that the value is quite different than that obtained from our whistler measurements $\left(-17 \times 10^{7} \mathrm{el} \mathrm{cm}^{-2} \mathrm{~s}^{-1}\right)$ at $L=2.9$ between DoY 217 and DoY 218 (Table 1). It must be considered however, that the two values obtained from whistler and FLR observations are not directly comparable because they refer to regions with very large longitudinal separation: $140^{\circ}$. Also, as pointed out by Dent et al. (2006), the value of the refilling can be significantly dependent on the local time when it is calculated; in particular, whistler measurements refer to late afternoon hours when plasma drainage from the plasmasphere to the ionosphere may occur. 

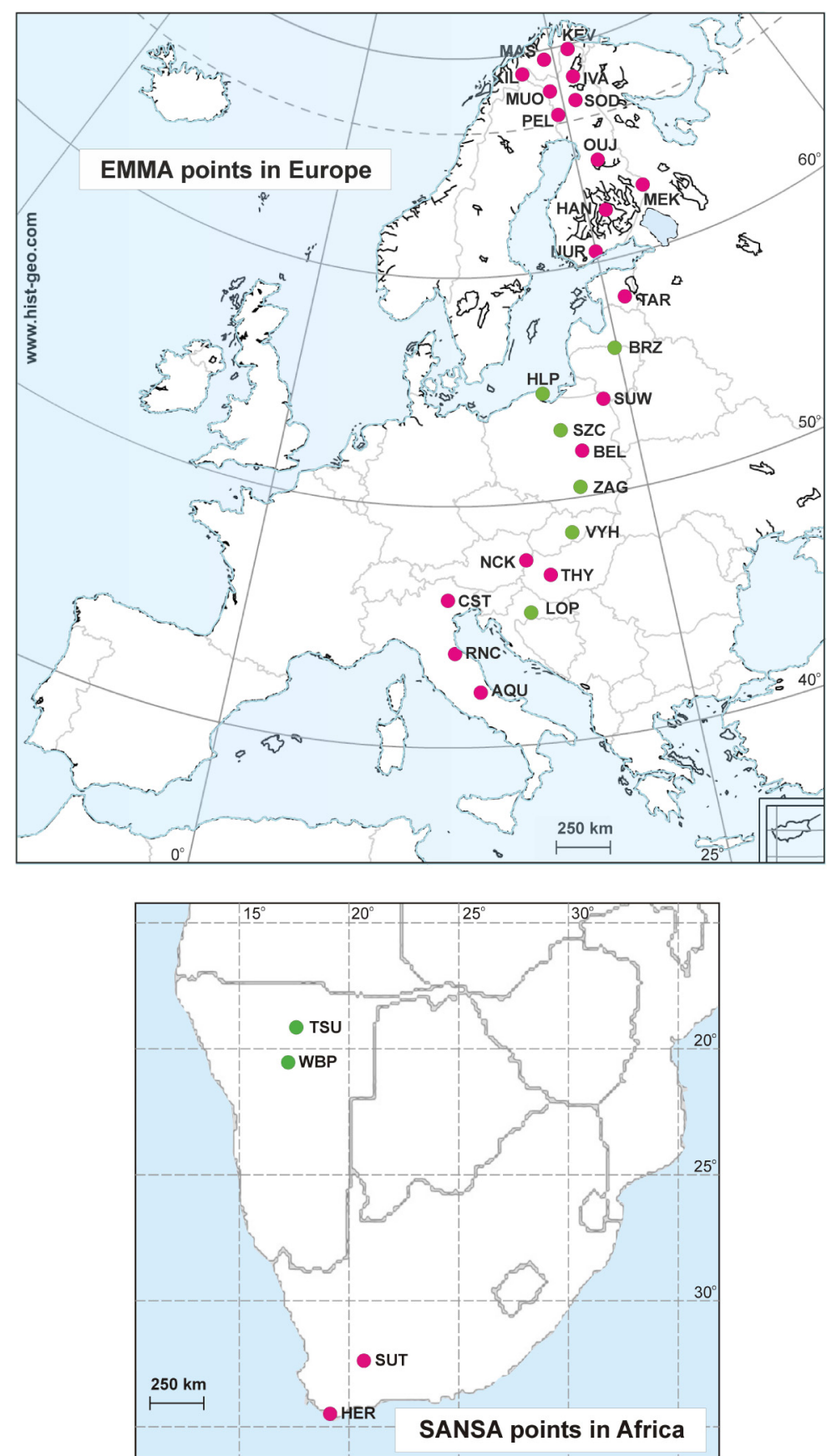

Fig. 3. Top panel: EMMA stations across Europe. The new stations installed in PLASMON (green dots) are Lonjsko Polje (LOP), Vyhne (VYH), Zagorzyce (ZAG), Szczechowo (SZC), Hel (HLP), and Birzai (BRZ). Bottom panel: SANSA stations in South Africa. The new stations installed in PLASMON are Waterberg Plateau Park (WBP) and Tsumeb (TSU). Geographic coordinates are shown.

We also evaluated for different $L$-shells the daytime refilling rate from the ionosphere during each of the three days preceeding the storm and each of the four days of recovery (Table 2). The evaluation was made in the 0400-1400 UT interval ( $\sim 06-16$ LT). The values of Table 2 indicate a clear increase of $(\Phi$ with increasing $L$, and a decrease of $\Phi$ in the recovery phase which might be attributed to a reduced plasma supply from the ionosphere whose ion content is usually reduced during the early phase of the magnetic storms recovery. The values are in line with the estimates of $10-50 \times$ $10^{7} \mathrm{amu} \mathrm{cm} \mathrm{cm}^{-2}$ obtained by Obana et al. (2010) at $L=2.3-3.8,57 \times 10^{7} \mathrm{amu} \mathrm{cm}{ }^{-2} \mathrm{~s}^{-1}(L=2$, Chi et al. 2000), and $30 \times 10^{7} \mathrm{el} \mathrm{cm}^{-2} \mathrm{~s}^{-1}(L \sim 3.7$, Park 1970).
The results of Figure 5 also show an anomalous sharp increase of the resonant period and of the inferred plasma mass density in the late evening of some days (e.g., on DoY 212, $214,218,219)$. An anomalously high resonant period could arise from the formation of a quarter-wave mode standing wave when one end of the field line is sunlit and the other end is in darkness (Obana et al. 2008), or from a low ionospheric conductivity in both hemispheres (Ozeke \& Mann 2005). In either case the equatorial plasma mass density should be inferred using proper boundary conditions different from the standard assumption of perfect wave reflection at both hemispheres. For this reason we excluded these late evening values from the analysis of the daytime plasma refilling rates. 
J. Lichtenberger et al.: First results from the PLASMON
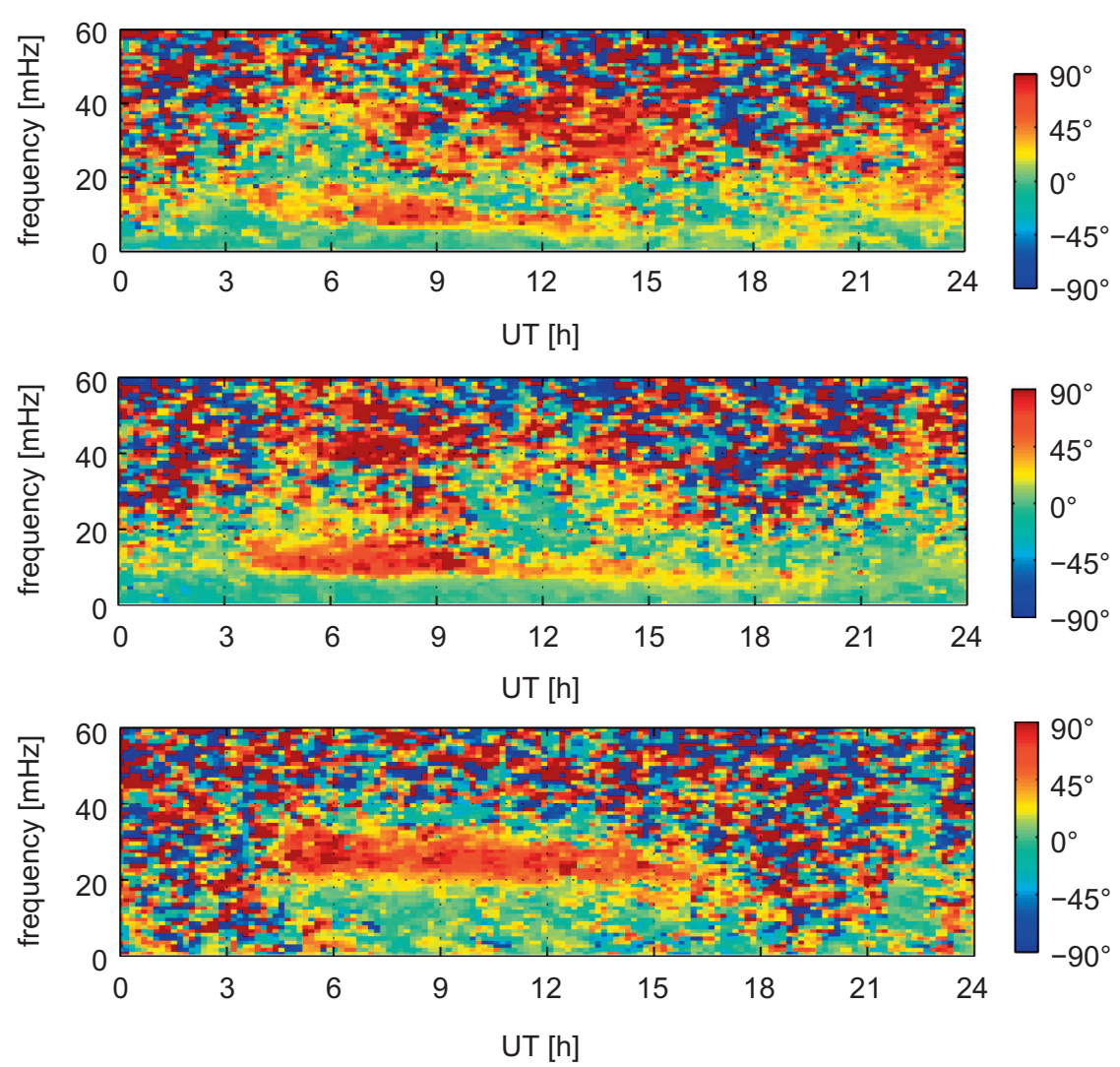

Fig. 4. Cross phase spectra for three station pairs, from top to bottom MEK-NUR $(L=3.7)$, NUR-TAR $(L=3.2)$, SUW-BEL $(L=2.4)$, respectively, Aug 1, 2010.
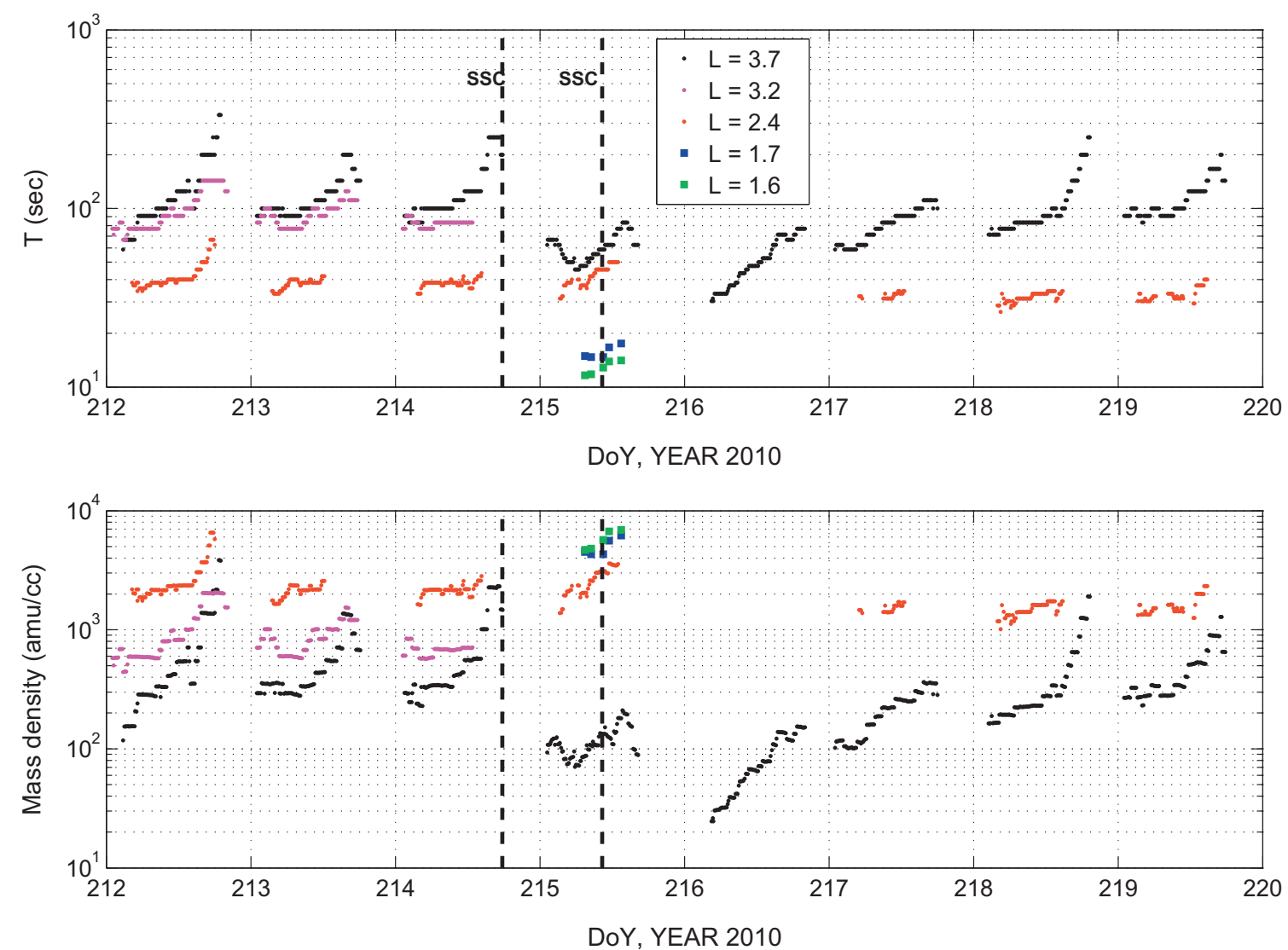

Fig. 5. FLR periods (upper panel) detected by FLRID and inferred equatorial plasma mass densities obtained by FLRINV (lower panel) at five station pairs (MEK-NUR, $L=3.7$; NUR-TAR, $L=3.2$; SUW-BEL, $L=2.4$; CST-RNC, $L=1.7$; RNC-AQU, $L=1.6$ ) over Aug 1-8, 2010. SSCs are marked by dashed vertical lines. 
Table 2. Daytime upward plasma flux across the 1000-km level for different flux tubes.

\begin{tabular}{cccc}
\hline \hline $\begin{array}{c}\text { Day of 2010 } \\
(0400-1400 \text { UT) }\end{array}$ & & $\Phi\left(10^{7} \mathrm{amu} \mathrm{cm}^{-2} \mathrm{~s}^{-1}\right)$ \\
\cline { 2 - 4 } & $L=2.4$ & $L=3.2$ & $L=3.7$ \\
\hline 1 August (DoY 212) & $15 \pm 6$ & $47 \pm 8$ & $80 \pm 6$ \\
2 August (DoY 213) & $23 \pm 7$ & $49 \pm 8$ & $42 \pm 7$ \\
3 August (DoY 214) & $7 \pm 5$ & $21 \pm 8$ & $57 \pm 7$ \\
5 August (DoY 216) & - & - & $13 \pm 9$ \\
6 August (DoY 217) & $8 \pm 8$ & - & $41 \pm 3$ \\
7 August (DoY 218) & $16 \pm 4$ & - & $24 \pm 4$ \\
8 August (DoY 219) & $9 \pm 4$ & - & $43 \pm 7$ \\
\hline
\end{tabular}

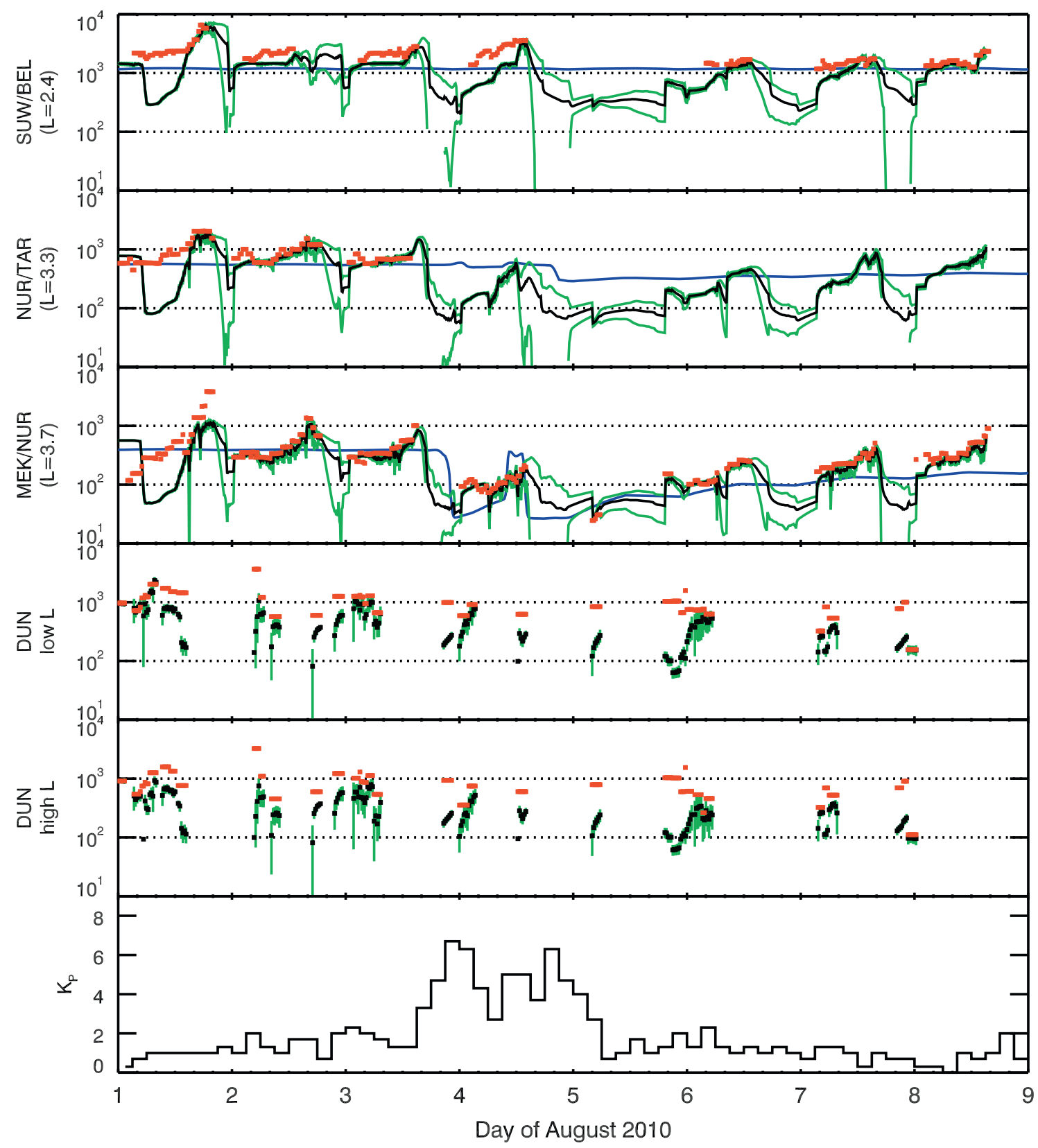

Fig. 6. Data assimilation result for the storm on August 3, 2010. The bottom panel shows the $K_{\mathrm{p}}$ index for the event, showing high activity level beginning near the end of August 3 and continuing into August 5. The top three panels show results for the three FLR station pairs, SUW/BEL $(L=2.4)$ in panel 1, NUR/TAR $(L=3.3)$ in panel 2, and MEK/NUR $(L=3.7)$ in panel 3. In those panels the blue trace represents the plasma density obtained from a reference model using a electric field derived from the $K_{\mathrm{p}}$ index. Panels 4 and 5 are results for the Dunedin VLF station. Panel 4 is the density at the innermost $L$-shell of a VLF whistler group, and panel 5 is at the outermost $L$-shell. All densities are in $\mathrm{cm}^{-3} \mathrm{The}$ red points are the observations. In the case of Dunedin each point represents a different $L$-shell range, nominally in the $L=3$ to $L=4$ range. The black traces represent the average assimilation output and the green traces the uncertainty around it. For the FLR stations, which map to a fixed location, assimilation output can be obtained even when no observations are available. For the VLF observations it is not useful to obtain assimilation output without observations because each observations is at a different $L$-shell. 


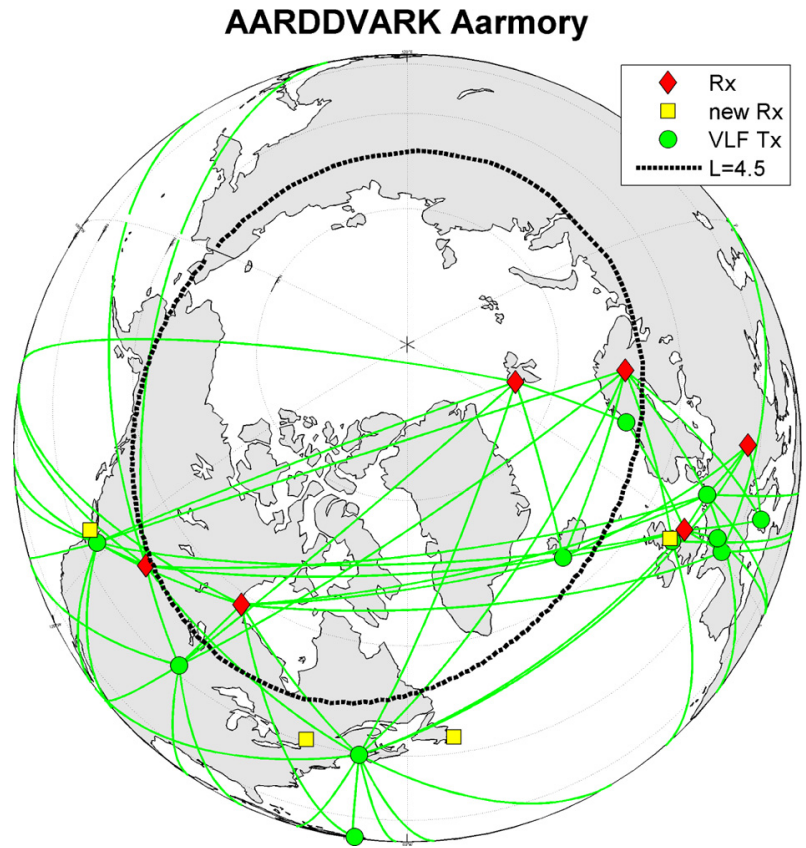

Fig. 7. The Northern Hemisphere AARDDVARK network. The green circles are the VLF transmitters, the red diamonds are the AARDDVARK receivers. The green lines show the great circle paths between the transmitters and the receivers. The dashed black oval shows the magnetic latitude of the footprint of the expected quiettime average plasmapause position in terms of the McIlwain $L$-shell parameter (in this case $L=4.5$ ). The four new stations installed in PLASMON are Forks (Seattle, USA), Ottawa and St. John's (both in Canada), and Eskdalemuir (Scotland).

\section{Data assimilative modeling of Earth's plasmasphere}

The use of data assimilation in space physics is still in its infancy. Data assimilation methods are used in ionospheric modeling (Bust et al. 2004; Bust \& Crowley 2007) and are beginning to be used in radiation belt modeling as well (Fuller-Rowell et al. 2006; Koller et al. 2007; Kondrashov et al. 2007; Reeves et al. 2012), and one example exists of using it to constrain a ring current model using global ENA images (Nakano et al. 2008). The relatively slow adoption of data assimilation for magnetospheric physics may be connected to the relative sparsity of observations.

A variety of plasmasphere models are used as drivers to existing ring current and radiation belt models to compute the loss processes (e.g., Fok et al. 1991, 2001; Friedel et al. 2002). Even the radiation belt models and ring current models that have been run under a data assimilation scheme do not include data assimilation on the plasmasphere but for example simply use an electric field parametrized by geomagnetic activity index such as $K_{\mathrm{p}}$.

Under the PLASMON project we have developed a data assimilation model of the plasmasphere based on the Dynamic Global Core Plasma Model (DGCPM) (Ober \& Horwitz 1997), and an Ensemble Kalman Filter (EnKF), for use with groundbased plasma density observations. The data assimilation model from PLASMON has not yet been published, but another paper (Jorgensen et al. 2011) details some early work toward the data assimilation. In this project we expand this capability in several ways, including adding the ability to use more data sources, adding composition information and the relevant refilling and loss rates, adding information about the field-aligned distribution of plasma, and improving the parametrization of the electric fields.

Figure 6 shows a assimilation result for August 3, 2010 storm. The bottom panel shows the $K_{\mathrm{p}}$ index which shows the storm main phase on August 3 and 4. The top three panels show magnetometer FLR observations and assimilation results, and the 4th and 5th panel show VLF whistler data and assimilation results. In the top three panels the blue curve is a reference model run without data assimilation, based solely on the $K_{\mathrm{p}}$ index. It shows that around $L=3.7$ the plasmasphere depleted at approximately 0 UT on August 4. At $L=3.3$ the depletion happened one day later and was not as large. The red points are observations. In the top three panels we see good coverage of data on the dayside although NUR/TAR did not observe FLRs after the onst. In the 4th and 5th panel the observations appear more scattered. This is because VLF stations do not measure density at a fixed $L$-shell but rather at a range of $L$ shells, with the range different for each whistler group. In this plot we elected to plot the density at the inner and outer $L$-shell, and thus the time series will generally not represent a single $L$ shell. Dunedin is at $L=3.5$, and the range of observations is from $L=3$ to $L=4$, approximately.

In all panels the black trace represents the assimilation output at the observations location, and the green traces represent the uncertainty. In the case of the FLR observations we could obtain model output even between observations because the FLR stations map to a fixed location. In the case of the VLF observations each data point is associated with a unique $L$-shell and thus it does not make sense to sample the assimilation model during times when no observations are available.

In general the agreement is good between the assimilation and the observations. A notable exception is August 1, which we will return to in a moment. We used observation uncertainties of $20-30 \%$ in the assimilation. One expected feature of data assimilation is that during time-intervals when there are no observations the uncertainty increases. This can be seen throughout the plot, but is perhaps most evident on August 5. Following one group of observations at approximately 4 UT at Dunedin and MEK/NUR there are no observations for $12 \mathrm{~h}$. During that time the model is running open loop and the uncertainty increases. As observations become available the uncertainty drops, and the model steps in the direction of the observations. In the case of August 5 that step is rather large.

On August 1 the agreement between observations and model is poor. This is almost certainly because of the initial conditions of the model used. We used a fully saturated plasmasphere as the initial condition at 0 UT on August 1. In order to agree with the observations the plasmasphere needed to be severely eroded and that takes at least $12-24 \mathrm{~h}$ to accomplish. During that time the assimilation model simply does not have the degrees of freedom to obtain good agreement with observations.

\section{Modeling REP losses from the radiation belts using the AARDDVARK network}

During a geomagnetic storm the length of time during which space assets are in danger is determined by the efficiency of the loss mechanisms, particularly through REP into the atmosphere. The primary mechanism for this precipitation is the interaction of several wave modes with resonant electrons, 


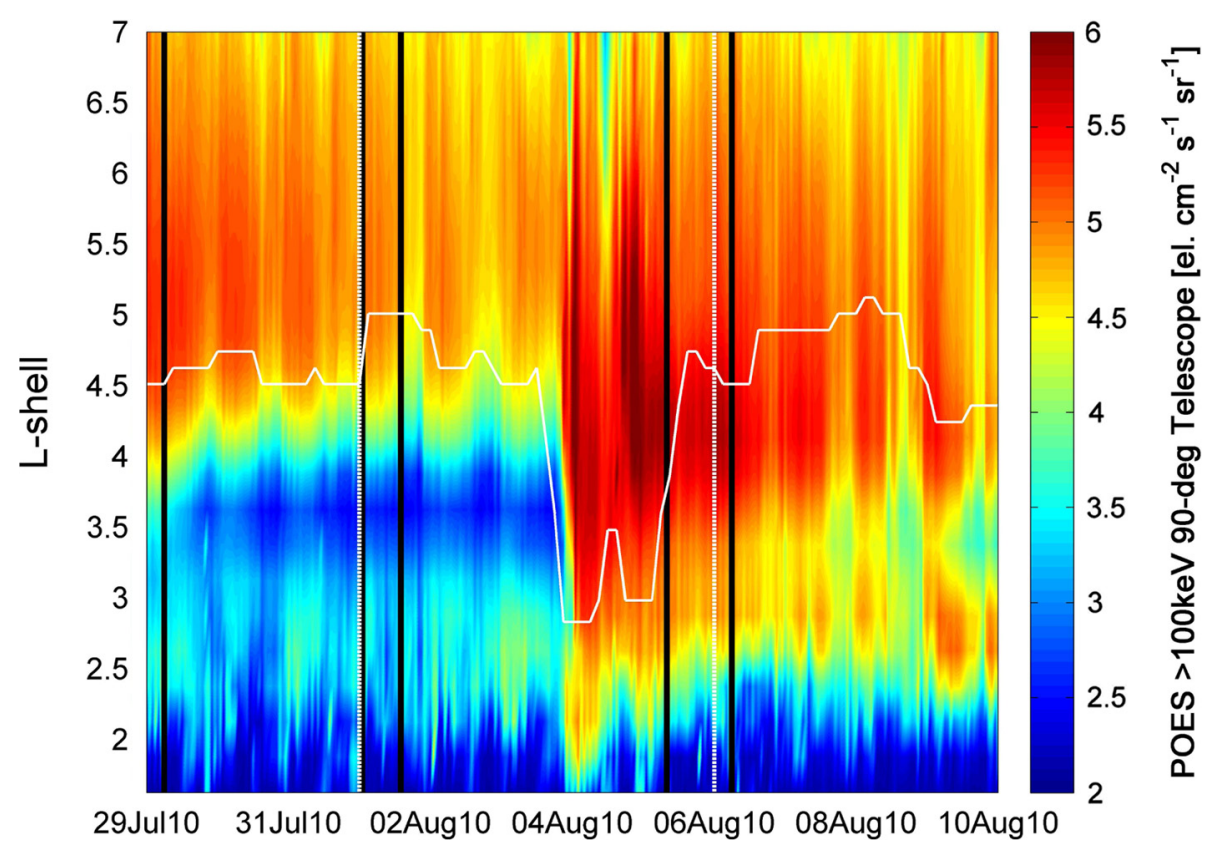

Fig. 8. POES (NOAA-17 to 19 and MetOp02) Space Environment Monitor (SEM-2) $>100 \mathrm{keV}$ quasi-trapped electron fluxes over 29 Jul-10 Aug, 2001. Vertical white dotted lines denote the study interval of interest. The white line shows the calculated position of plasmapause based on $12 \mathrm{~h}$ maximum $K_{\mathrm{p}}$ index value (Moldwin et al. 2002).

which leads to scattering into the atmospheric loss cone. The nature of the wave activity and the interactions between the waves and radiation belt particles are strongly governed by the properties of the plasmasphere. We use the assimilative model of the plasmasphere to identify regions where plasmaspheric structures such as the regions occurring on, inside, and outside of the plasmaspause and/or composition changes are likely to result in enhanced electron losses. We monitor the occurrence and properties of REP using the ground-based AARDDVARK network (Clilverd et al. 2009). The Northern Hemisphere AARDDVARK map (Fig. 7) shows all of the stations including those completed as part of the PLASMON project: Forks (Seattle, USA), Ottawa, and St John's (Canada) and Eskdalemuir (Scotland). With the completion of the AARDDVARK network in the Northern Hemisphere we are now in a strong position to monitor the electron precipitation coming from within, on, and outside of, the plasmapause. The dashed circle on the plot shows the $L$-shell contour at $L=4.5$, which represents the average quiet-time location of the plasmapause.

Figure 8 shows electron flux measurements for the August $2010 \mathrm{ssc} / \mathrm{storm}$ interval from the MEPED instrument which is part of the Space Environment Monitor-2 (SEM-2) experiment carried on-board the POES spacecraft. These measurements have been zonally averaged after correction for low-energy proton contamination (following Lam et al. 2010) and include observations made by all six spacecraft which carried the SEM-2 and were in orbit at that time (NOAA-15 through to -19 and MetOp-02). The electron fluxes shown in Figure 8 are $>100 \mathrm{keV}$ quasi-trapped electron fluxes during the period July 29 through to August 10, with the studied period (August 1-6, 2010) marked with white dashed lines. The plot shows the fluxes in a logarithmic color scale from $10^{2}-10^{6} \mathrm{el} \mathrm{cm}^{-2} \mathrm{~s}^{-1} \mathrm{sr}^{-1}$. The fluxes are being measured close to the drift-loss cone. Thus these fluxes, while trapped, are only slightly above the loss cone in pitch angle space. It is these electron fluxes which are most likely to be scattered into the bounce loss cone during any geomagnetic activity which enhances wave-particle interactions, and thus these fluxes represent the electrons available to be lost into the atmosphere. The fluxes show an increase from background levels of about $3 \times 10^{4} \mathrm{el} \mathrm{cm}^{-2} \mathrm{~s}^{-1} \mathrm{sr}^{-1}$ to $1 \times 10^{6} \mathrm{el} \mathrm{cm}^{-2} \mathrm{~s}^{-1} \mathrm{sr}^{-1}$ during the first SSC on August 3rd, with high flux levels observed at much lower $L$-shells than before the storm period. The calculated position of the plasmapause from the empirical plasmapause model of Moldwin et al. (2002), which uses a $12-\mathrm{h} K_{\mathrm{p}}$ maximum value, is shown as a white line. The highest $>100 \mathrm{keV}$ flux levels occur outside of the Moldwin et al. (2002) plasmapause. Initially the plasmapause is located at about $L=4.5$, but during the storm it moves into about $L=3$ for about one day. During this period high fluxes occur at low $L$-shells as a result of the inward movement of the plasmapause, and then remain elevated at those $L$-shells for several days after the plasmapause has recovered back to $L=4.5$. Similar links between plasmaspheric dynamics and the apparent motion of the radiation belt location have been reported previously (Baker et al. 2004; Rodger et al. 2007). After August 4 high fluxes of quasi-trapped electrons will be subject to wave-particle interactions occurring inside, on, and outside the plasmapause. PLASMON aims to refine this picture, by accurately locating the plasmapause, identifying the density levels and composition (which influence wave-particle interactions), and measuring the electron precipitation that actually occurs.

Figure 9 shows an example of how the AARDDVARK VLF data responds to the precipitation occurring in this time period. We have analyzed the observations made by the AARDDVARK receiver at Churchill (Canada) of the transmissions originating from the US Navy communications station in North Dakota (call sign NDK). Initially, we analyzed the received amplitude on days which were geomagnetically quiet and not affected by significant electron precipitation. This provides a statistically generated quiet day curve (QDC) for the normal diurnal amplitude variation, including also a standard deviation to represent the experimental uncertainties in the 
J. Lichtenberger et al.: First results from the PLASMON
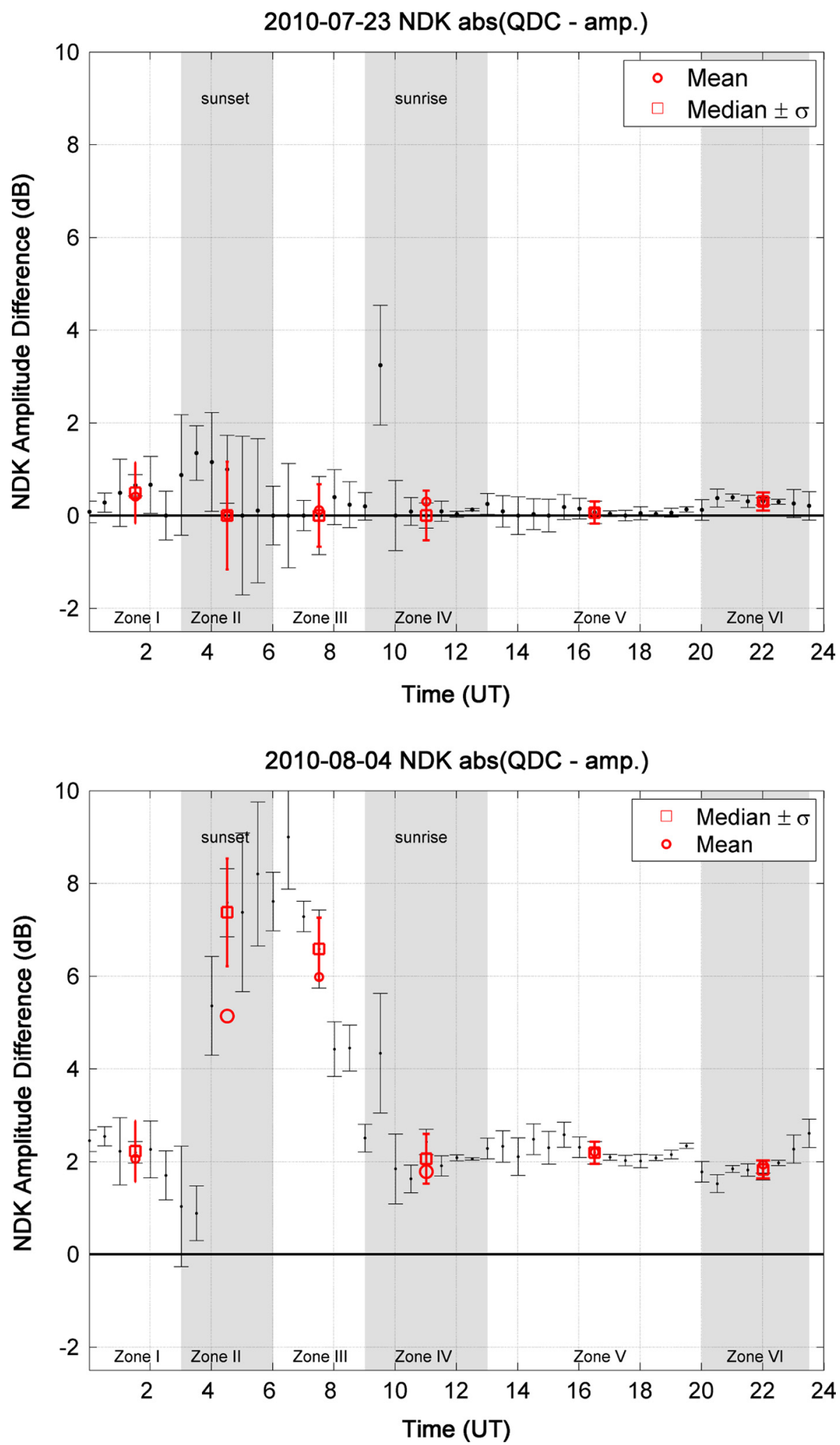

Fig. 9. Top panel: The change in amplitude of the North Dakota US Navy VLF transmitter signal received at Churchill (Canada) on a relatively undisturbed day (July 23, 2010) after the removal of a "quiet day curve" from the signal. Bottom panel: The change in amplitude of the North Dakota US Navy VLF transmitter signal received at Churchill (Canada) on August 4, 2010, after the removal of a quiet day curve from the signal. The red dots with error bars represent the mean and standard deviation of the amplitude perturbations for each of the UT time zones identified by the shading, including those periods which involve sunrise and sunset on the propagation path.

QDC generation. Even in quiet times the received VLF amplitudes are most variable during the sunrise and sunset periods, and throughout the night, such that the uncertainty is higher for these time periods (marked Zones II through to IV in the Figure). The top panel of Figure 9 shows the change in the received NDK amplitude (i.e., change relative to the QDC) 
on a relatively quiet day (July 23, 2010). As expected, there is little evidence of significant ionospheric disturbances on this day, as the mean and median amplitude differences are close to zero, particularly during the midday period (Zone VI) where the Sun dominates the $D$-region ionosphere and hence is the main factor influencing quiet time VLF propagation.

The lower panel of Figure 9 shows the change in the received NDK amplitude on a disturbed day during our study period (August 4, 2010). This clearly exhibits large amplitude perturbations relative to the QDC. There is evidence of precipitation across the entire day, with a near constant offset of $\sim 2 \mathrm{~dB}$ in the Sun-lit periods and much larger amplitude changes when the $D$-region is dominated by nighttime conditions (Zones II and IIII, i.e., 3-8UT). However, while the amplitude changes are larger during the day than during the night, VLF propagation tends to be more sensitive to precipitation during the night due to the more tenuous $D$-region (i.e., Rodger et al. 2010, 2012). The next step is for us to model the VLF propagation conditions, and then estimate the precipitating flux levels for different times of day in order to reproduce the amplitude perturbations using the approaches outlined in Rodger et al. (2012). This is a necessary part of our plan to achieve one of the goals of PLASMON, modeling observed REP losses from the radiation belts and relating those to plasmaspheric structures.

Figure 9 clearly shows that the AARDDVARK data are responding to the additional electron precipitation occurring into the lower ionosphere on this day. By comparison with Figure 8 we can also see that the VLF propagation path observations presented in Figure 9 (where the path ranges from $3<L<7$ ) are only influenced by precipitation from outside of the plasmasphere on August 4. This is consistent with loss mechanisms such as chorus waves, which are known to occur outside of the plasmaspause, and to be enhanced during periods of high geomagnetic disturbance (Meredith et al. 2012). Using the AARDDVARK data to model the effect of electron precipitation fluxes on the North Dakota to Churchill path during this intense storm period will allow us to quantify the chorusinduced loss mechanism. By analogy, we will also be able to use AARDDVARK data during the later stages of the geomagnetic storm, from VLF propagation paths that range from $3<L<4.5$. This will allow us to compare and quantify electron precipitation fluxes resulting from processes from inside the plasmapause, such as plasmaspheric hiss-induced loss mechanisms (Rodger et al. 2007).

\section{Conclusions and future work}

During the first 24 months of the PLASMON project, we have extended our ground-based VLF and ULF networks, installing three new stations in AWDANet, four new stations in AARDDVARK, and eight new stations in our ULF network (six in the Europen EMMA and two in the Southern African SANSA network). The extended networks are used to achieve the objective of the project. We have developed an algorithm that allows us to retrieve electron density profiles automatically and we have implemented the algorithm on GPU-based processing units and we are working on to reach a quasi-real-time mode of operation of AWDANet.

An automated algorithm for identification of FLSs, FLRID has been developed in PLASMON, which provides the input for the automatic inversion procedure (FLRINV).
The assimilative model of the plasmasphere is the central core of the project. It is based on the Dynamic Global Core Plasma Model and a Ensemble Kalman Filter. We have started to test the assimilation using density data from our two groundbased networks (AWDANet and EMMA).

The third ground-based network (AARDDVARK) is used to contrast the plasmasphere model through comparison of REP losses. In this paper we have illustrated the combined use of these resources with preliminary investigation of a storm interval over August 1-6, 2010. Results include estimation of electron and ion mass densities and coupling rates before and during the storm, and changes in quasi-trapped electron fluxes near their scattering point into the loss cone, forming REP detected over VLF paths on the ground.

Acknowledgements. The research leading to these results has received funding from the European Union Seventh Framework Programme [FP7/2007-2013] under Grant Agreement No. 263218

\section{References}

Berube, D., M.B. Moldwin, and J.M. Weygand, An automated method for the detection of field line resonance frequencies using ground magnetometer techniques, J. Geophys. Res., 108, 1348, 2003.

Baker, D.N., S. Kanekal, X. Li, S.P. Monk, J. Goldstein, and J.L. Burch, An extreme distortion of the Van Allen belt arising from the 'Hallowe'en' solar storm in 2003, Nature, 432, 879-880, 2004.

Berube, D., M.B. Moldwin, and M. Ahn, Computing magnetospheric mass density from field line resonances in a realistic magnetic field geometry, J. Geophys. Res., 111, A08206, 2006.

Bust, G.S., and G. Crowley, Tracking of polar cap ionospheric patches using data assimilation, J. Geophys. Res., 112, A05307, 2007.

Bust, G., T. Garner, and T.L. Gaussiran, Ionospheric data assimilation three dimensional (IDA3D): a global, multi-sensor, electron density specification algorithm, J. Geophys. Res., 109, A11313, 2004.

Chi, P.J., C.T. Russell, S. Musman, W.K. Peterson, G. Le, V. Angelopoulos, G.D. Reeves, M.B. Moldwin, and F.K. Chun, Plasmaspheric depletion and refilling associated with the September 25, 1998 magnetic storm observed by ground magnetometers at L = 2, Geophys. Res. Lett., 27, 633-636, 2000.

Clilverd, M.A., C.J. Rodger, N.R. Thomson, J.B. Brundel, T. Ulich, J. Lichtenberger, N. Cobbett, A.B. Collier, F.W. Menk, A. Seppälä, P.T. Verronen, and E. Turunen, Remote sensing space weather events: Antarctic-Arctic radiation-belt (dynamic) deposition-VLF Atmospheric Research Konsortium network, Space Weather, 7, S04001, 2009.

Collier, A.B., S. Bremner, J. Lichtenberger, J.R. Downs, C.J. Rodger, P. Steinbach, and G. McDowell, Global lightning distribution and whistlers observed at Dunedin, New Zealand, Ann. Geophys., 28, 499-513, 2010.

Darrouzet, F., J. De Keyser, and V. Pierrard, The Earth's plasmasphere: a CLUSTER and IMAGE perspective, Springer, New York, springer.com, 2009.

Dent, Z.C., I.R. Mann, J. Goldstein, F.W. Menk, and L.G. Ozeke, Plasmaspheric depletion, refilling, and plasmapause dynamics: a coordinated ground-based and IMAGE satellite study, J. Geophys. Res., 111, A03205, 2006.

Evensen, G., The ensemble Kalman filter: theoretical formulation and practical implementation, Ocean Dyn., 53 (4), 343-367, 2003.

Fok, M.C., J.U. Kozyra, A.F. Nagy, and T.E. Cravens, Lifetime of ring current particles due to coulomb collisions in the plasmasphere, J. Geophys. Res., 96 (A5), 7861-7867, 1991. 
Fok, M.C., R.A. Wolf, R.W. Spiro, and T.E. Moore, Comprehensive computational model of Earth's ring current, J. Geophys. Res., 106 (A5), 8417-8424, 2001.

Friedel, R.H.W., G.D. Reeves, and T. Obara, Relativistic electron dynamics in the inner magneto sphere - a review, J. Atmos. Terr. Phys., 64, 265-282, 2002.

Fuller-Rowell, T., E. Araujo-Pradere, C. Minter, M. Codrescu, P. Spencer, D. Robertson, and A.R. Jacobson, US-TEC: a new data assimilation product from the Space Environment Center characterizing the ionospheric total electron content using real-time GPS data, Radio Sci., 41, RS6003, 2006.

Green, A.W., E.W. Worthington, L.N. Baransky, E.N. Fedorov, N.A. Kurneva, V.A. Pilipenko, D.N. Shvetzov, A.A. Bektemirov, and G.V. Philipov, Alfvén field line resonances at low latitudes $(L=1.5), J$. Geophys. Res., 98, 15693-15699, 1993.

Heilig, B., S. Lotz, J. Vero, P. Sutcliffe, K.P.J. Reda, and T. Raita, Empirically modelled pc3 activity based on solar wind parameters, Ann. Geophys., 28, 1703-1722, 2010.

Jorgensen, A., D. Ober, J. Koller, and R.H.W. Friedel, Specification of the earth's plasmasphere with data assimilation, Adv. Space Res., 47, 2152-2161, 2011.

Koller, J., Y. Chen, G.D. Reeves, R.H. Friedel, T.E. Cayton, and J.A. Vrugt, Identifying the radiation belt source region by data assimilation, J. Geophys. Res., 112, A06244, 2007.

Kondrashov, D., D.Y. Shprits, M. Ghil, and R. Thornet, A Kalman filter technique to estimate relativistic electron lifetimes in the outer radiation belt, J. Geophys. Res., 112, A10227, 2007.

Lam, M.M., R.B. Horne, N.P. Meredith, S.A. Glauert, T. Moffat-Griffin, and J.C. Green, Origin of energetic electron precipitation $>30 \mathrm{keV}$ into the atmosphere, J. Geophys. Res., 115, A00F08, 2010.

Lichtenberger, J., A new whistler inversion method, J. Geophys. Res., 114, A07222, 2009.

Lichtenberger, J., C. Ferencz, L. Bodnár, D. Hamar, and P. Steinbach, Automatic whistler detector and analyzer system: automatic whistler detector, J. Geophys. Res., 113, A12201, 2008.

Lichtenberger, J., C. Ferencz, D. Hamar, P. Steinbach, C.J. Rodger, M.A. Clilverd, and A.B. Collier, The automatic whistler detector and analyzer (AWDA) system: implementation of the analyzer algorithm, J. Geophys. Res., 115, A12214, 2010.

Meredith, N.P., R.B. Horne, A. Sicard-Piet, D. Boscher, K.H. Yearby, W. Li, and R.M. Thorne, Global model of lower band and upper band chorus from multiple satellite observations, $J$. Geophys. Res., 117, A10225, 2012.

Moldwin, M.B., L. Downward, H.K. Rassoul, R. Amin, and R.R. Anderson, A new model of the location of the plasmapause: CRRES results, J. Geophys. Res., 107, 1339-1348, 2002.

Nakano, S., G. Ueno, Y. Ebihara, M.-C. Fok, S. Ohtani, P.C. Brandt, D.G. Mitchell, K. Keika, and T. Higuchi, A method for estimating the ring current structure and the electric potential distribution using energetic neutral atom data assimilation, J. Geophys. Res., 113, A05208, 2008

Obana, Y., F.W. Menk, M.D. Sciffer, and C.L. Waters, Quarter-wave modes of standing Alfvén waves detected by cross-phase analysis, J. Geophys. Res., 113, A08203, 2008.
Obana, Y., F.W. Menk, and I. Yoshikawa, Plasma refilling rates for $L=2.3-3.8$ flux tubes, J. Geophys. Res., 115, A03204, 2010.

Ober, D.M., and J.L. Horwitz, Formation of density troughs embedded in the outer plasmasphere by subauroral ion drift events, J. Geophys. Res., 102, 14595-14602, 1997.

Ozeke, L.G., and I.R. Mann, High and low ionospheric conductivity standing 478 guided Alfvén wave eigenfrequencies: a model for plasma density mapping, J. Geophys. Res., 115, A03204, 2005.

Park, C.G., Whistler observations of the interchange of ionization between the ionosphere and the protonosphere, J. Geophys. Res., 75, 4249-4260, 1970.

Park, C.G., Methods to determine electron concentrations in the magnetosphere from nose whistlers, Technical report 3454-1, Radioscience Laboratory, Stanford Electronics Laboratories, Stanford University, Stanford, California, 1972.

Rodger, C.J., M.A. Clilverd, N.R. Thomson, R.J. Gamble, A. Seppälä, E. Turunen, N.P. Meredith, M. Parrot, J.A. Sauvaud, and J.-J. Berthelier, Radiation belt electron precipitation into the atmosphere: recovery from a geomagnetic storm, J. Geophys. Res., 112, A11307, 2007.

Rodger, C.J., M.A. Clilverd, A. Seppälä, N.R. Thomson, R.J. Gamble, M. Parrot, J.A. Sauvaud, and T. Ulich, Radiation belt electron precipitation into the atmosphere: recovery from a geomagnetic storm, J. Geophys. Res., 115, A11320, 2010.

Reeves, G.D., Y. Chen, G.S. Cunningham, R.W.H. Friedel, M.G. Henderson, V.K. Jordanova, J. Koller, S.K. Morley, M.F. Thomsen, and S. Zaharia, Dynamic radiation environment assimilation model: DREAM, Space Weather, 10, S03006, 2012.

Rodger, C.J., M.A. Clilverd, A.J. Kavanagh, C.E.J. Watt, P.T. Verronen, and T. Raita, Contrasting the responses of three different ground-based instruments to energetic electron precipitation, Radio Sci., 47 (2), RS2021, 2012.

Sazhin, S.S., M. Hayakawa, and K. Bullough, Whistler diagnostics of magnetospheric parameters: a review, Ann. Geophys., 10, 293308, 1992.

Singer, H.J., D.J. Southwood, R.J. Walker, and M.G. Kivelson, Alfvén wave resonances in a realistic magnetospheric magnetic field geometry, J. Geophys. Res., 86, 4589-4596, 1981.

Tsyganenko, N.A., A model of the near magnetosphere with a dawndusk asymmetry 1. Mathematical structure, J. Geophys. Res., 107 (A8), SMP 12-1-SMP12-5, 2002a.

Tsyganenko, N.A., A model of the near magnetosphere with a dawndusk asymmetry 2 . Parameterization and fitting to observations, $J$. Geophys. Res., 107 (A8), SMP 10-1-SMP 10-17, 2002 b.

Vellante, M., and M. Förster, Inference of the magnetospheric plasma mass density from field line resonances: a test using a plasmasphere model, J. Geophys. Res., 111, A11204, 2006.

Vellante, M., H. Lühr, T.L. Zhang, V. Wesztergom, U. Villante, M. DeLauretis, A. Piancatelli, M. Rother, K. Schwingenschuh, W. Koren, and W. Magnes, Ground/satellite signatures of field line resonance: a test of theoretical predictions, J. Geophys. Res., 109, A06210, 2004.

Waters, C.L., F.W. Menk, and B.J. Fraser, The resonance structure of low latitude pc3 geomagnetic pulsations, Geophys. Res. Lett., 18, 2283-2296, 1991.

Cite this article as: Lichtenberger J, Clilverd M, Heilig B, Vellante M, Manninen J, et al.: The plasmasphere during a space weather event: first results from the PLASMON project. J. Space Weather Space Clim., 2013, 3, A23. 